\title{
Absolute Neutrophil Count Predicts Postoperative Prognosis in Mass-forming Intrahepatic Cholangiocarcinoma
}

\author{
AKIRA WATANABE ${ }^{1}$, NORIHUMI HARIMOTO ${ }^{1}$, KENICHIRO ARAKI $^{1}$, NORIO KUBO $^{1}$, \\ TAKAMICHI IGARASHI ${ }^{1}$, MARIKO TSUKAGOSHI ${ }^{1}$, NORIHIRO ISHII $^{1}$, \\ TAKAHIRO YAMANAKA ${ }^{1}$, TOMOHARU YOSHIZUMI ${ }^{2}$ and KEN SHIRABE ${ }^{1}$ \\ ${ }^{1}$ Department of Hepatobiliary and Pancreatic Surgery, \\ Gunma University Graduate School of Medicine, Maebashi, Japan; \\ ${ }^{2}$ Department of Surgery and Science, Graduate School of Medical Sciences, Kyushu University, Fukuoka, Japan
}

\begin{abstract}
Background/Aim: Intrahepatic cholangiocarcinoma (IHCC) is characterized by poor prognosis, and postoperative recurrence remains a problem. Thus, prognostic markers for IHCC are greatly needed. Recently, inflammatory factors were reported to be related to tumor progression and recurrence in various cancers. Therefore, the present study aimed to evaluate the prognostic ability of inflammatory factors. Materials and Methods: Forty-four patients with mass-forming IHCC ( $m$-IHCC) were retrospectively evaluated and the correlations between inflammatory markers, including neutrophil-tolymphocyte ratio and, modified Glasgow prognostic score, and patient survival were assessed. Results: The absolute neutrophil count (ANC) was significantly higher in the recurrence group than in the non-recurrence group $(p=0.00568)$ and the most significant prognostic factor by multivariate analysis. Poor recurrence-free survival (RFS; $p=0.00452)$ and cancer-specific survival (CSS; $p=0.0323$ ) were associated with high neutrophil levels. Moreover, neutrophil infiltration in the tumor site was positively correlated with ANC. Conclusion: ANC is associated with poor RFS, and could be used to predict recurrence in patients with m-IHCC.
\end{abstract}

Intrahepatic cholangiocarcinoma (IHCC) is characterized by poor prognosis, and its incidence is increasing globally (1). Although surgical therapy is the most effective approach for IHCC, the rate of postoperative recurrence has been reported to be greater than $60 \%$, and the 5 -year survival rate has been

Correspondence to: Norihumi Harimoto, Department of Hepatobiliary and Pancreatic Surgery, Gunma University Graduate School of Medicine, 3-39-22 Showa-machi, Maebashi Gunma 3718511, Japan. Tel: +81 272208800, Fax: +81 272208802, e-mail: nharimotoh1@gmail.com

Key Words: Absolute neutrophil count, intrahepatic cholangiocarcinoma, inflammation. reported to be $20-40 \%(1,2)$. For improving the prognosis of IHCC, it is necessary to identify mechanisms of progression and develop novel prognostic markers for IHCC.

Recently, inflammatory factors, such as the neutrophil-tolymphocyte ratio (NLR) and the modified Glasgow prognostic score (mGPS), were reported to be related to tumor progression, recurrence, and prognosis in various cancers (3, 4). In particular, NLR was reported to predict clinical prognostic outcomes in patients with colorectal and gastric cancer $(5,6)$, as well as postoperative prognosis in patients with IHCC $(7,8)$. Other factors that have been shown to be associated with IHCC occurrence and prognosis are hepatitis B or C virus infection $(9,10)$ and hepatolithiasis (11).

Many studies have demonstrated the critical role of inflammation, including inflammatory signaling pathways and infiltration of inflammatory cells, in the development and progression of IHCC (12-15). Thus, IHCC has a close relationship with inflammation and elucidating the underlying mechanism could help improve the prognosis of IHCC.

IHCC types can be divided into three categories according to the macroscopic growth pattern, the mass-forming (m-IHCC), the periductal-infiltrating, and the intraluminalgrowth type (16). The present study intended to evaluate the inflammatory status of m-IHCC. More specifically, inflammatory factors and their potential as predictive markers of m-IHCC were examined. Furthermore, tumor progression and inflammation were assessed in order to improve the prognosis of m-IHCC.

\section{Materials and Methods}

Patients and samples. The medical records of $44 \mathrm{~m}$-IHCC patients who underwent curative liver resection at our department and the Department of Surgery and Science, Kyushu University, between 2007 and 2016 were retrospectively evaluated. The age range of the patients was 46-88 years. Tumor staging was performed based on the Union for International Cancer Control tumor-node-metastasis (TNM) staging system (7th edition). Imaging examination, 
computerized tomography (CT), magnetic resonance imaging (MRI) and fluorodeoxyglucose-positron emission tomography (FDG-PET) was performed about 1 month before the start of therapy. Informed consent for treatment and analysis was obtained from all patients, and the study design was approved by our Clinical Ethics Committee; the clinical trial number of Gunma University is 2016103 (http://ciru.dept.showa.gunma-u.ac.jp/guidance/storage-sample/ list.html). Postoperative surveillance (5 years) was performed with blood examination every 1 month and CT or MRI imaging examination every 3 months.

Immunohistochemical staining. A 4- $\mu \mathrm{m}$ section was cut from paraffin blocks of IHCC samples. Each section was mounted on a silane-coated glass slide, deparaffinized, and soaked for $30 \mathrm{~min}$ at room temperature in $0.3 \% \mathrm{H} 2 \mathrm{O} 2 /$ methanol to block endogenous peroxidases. The sections were then heated in boiled water and Immunosaver (Nisshin EM, Tokyo, Japan) at $98^{\circ} \mathrm{C}$ for $45 \mathrm{~min}$. Nonspecific binding sites were blocked by incubating with Protein Block Serum-Free (DAKO, Santa Clara, USA) for $30 \mathrm{~min}$. A rabbit monoclonal anti-NF-kb antibody (CST, Danvers, USA) and a rabbit polyclonal anti-CD66b antibody (Abcam, Cambridge, UK) were applied at a dilution of $1: 100$ for $24 \mathrm{~h}$ at $4^{\circ} \mathrm{C}$. The primary antibody was visualized using the Histofine Simple Stain PO (M) Kit (Nichirei, Tokyo, Japan) according to the instruction manual. The chromogen 3,3ф-diaminobenzidine tetrahydrochloride was applied as a $0.02 \%$ solution containing $0.005 \% \mathrm{H}_{2} \mathrm{O}_{2}$ in $50 \mathrm{mM}$ ammonium acetate-citrate acid buffer ( $\mathrm{pH}$ 6.0). The sections were lightly counterstained with Mayer's hematoxylin and mounted. Negative controls were established by omitting the primary antibody, and no detectable staining was evident. Immunohistostaining results of all samples were evaluated by 2 observers by microscopy at $400 x$ every 3 fields. Cases with an average of 50 positive cells were judged to be CD66b-positive.

Statistical analysis. Significance was evaluated by performing the Student's $t$-test, analysis of variance, and Mann-Whitney $U$-test, and by determining Pearson's correlation coefficient. Survival curves were created by applying the Kaplan-Meier method and analyzed using the log-rank test. Clinical factors were examined by using both univariate and multivariate analyses on postoperative recurrence, via a logistic regression. The factors with a $p$-value $<0.1$ by univariate analysis were further evaluated by multivariate analysis. The cut-off value for the ANC was determined by using receiver operating characteristic curve analysis. All statistical analyses were performed using EZR (Saitama Medical Center, Jichi Medical University, Saitama, Japan), which is a graphical user interface for R (The R Foundation for Statistical Computing, Vienna, Austria) (16).

\section{Results}

Histopathological image of $m$-IHCC. Infiltration of inflammatory cells, especially neutrophils, in the tumor was observed in many cases. In addition, the inflammatory factor NF-kB was highly expressed in all $\mathrm{m}$-IHCC cases (Figure 1).

Association between postoperative recurrence and absolute neutrophil count (ANC) in m-IHCC patients who underwent resection. In this study, 44 patients underwent surgical therapy.
Postoperative recurrence was observed in 24 patients (55\%). Univariate and multivariate analyses were performed to determine significant predictors of postoperative recurrence, including inflammatory factors, by logistic regression methods (Table I). Multivariate analysis revealed that ANC (continuous variable) was an independent risk factor for postoperative recurrence $(\mathrm{HR}=4.520,95 \% \mathrm{CI}=1.46-14.0, p=0.00879)$. Therefore, ANC was selected as a prognostic factor and was further analyzed. The mean preoperative ANC was $4.51 \times 10^{3} / \mu \mathrm{l} \quad\left(\right.$ range $\left.=1.88-13.0 \times 10^{3} / \mu \mathrm{l}\right)$. The ANC was significantly higher in the recurrence group than in the recurrence-free group ( $p=0.00568$; Figure $2 \mathrm{~A}$ ). The optimal ANC cut-off was $3.945 \times 10^{3} / \mu$ l [area under the curve $(\mathrm{AUC})=0.721$, sensitivity $=0.800$, specificity $=0.750$; Figure 2B]. This cut-off value was utilized for subsequent analyses. The ANC had no correlations with clinicopathological findings (Table II). The ANC was significantly correlated with the NLR $(p=0.0112)$ and maximal standardized uptake value $\left(\mathrm{SUV}_{\text {max }}\right)(p=0.00089)$.

Correlation of the ANC and postoperative prognosis. With regard to postoperative prognosis, postoperative recurrencefree survival (RFS) and cancer-specific survival (CSS) were associated with the ANC. The high ANC group showed poorer RFS $(p=0.00045)$ and CSS $(p=0.03230)$ than the low ANC group (Figure 2C and D). A high ANC was independently associated with poor RFS in the multivariate analysis $(\mathrm{HR}=5.105,95 \% \mathrm{CI}=1.57-16.56, p=0.00678$; Table III), and the high ANC group had a tendency toward poor $\mathrm{CCS}$ in the multivariate analysis $(\mathrm{HR}=3.210,95 \% \mathrm{CI}=0.88$ 11.7, $p=0.07700$; Table IV).

To evaluate the relationship between inflammation and focal neutrophil agglutination in the tumor microenvironment, CD66b expression was investigated in resected samples. CD66b is a neutrophil activation marker (17). Neutrophil activation (CD66b positivity) in the local site had a positive correlation with the ANC ( $p=0.0335$; Figure 3$)$.

\section{Discussion}

In the present study, an $\mathrm{ANC} \geq 3.945 \times 10^{3} / \mu$ l was associated with postoperative $\mathrm{m}$-IHCC recurrence. The ANC could be a clinically useful prognostic marker for m-IHCC after surgical treatment.

Several previous studies reported a correlation between IHCC and inflammation with regard to cancer prognosis. Lin et al. reported that an NLR $>3$ was correlated with carcinoembryonic antigen (CEA), tumor size, TNM stage, and poor prognosis after hepatectomy (7). Additionally, Chen et al. demonstrated that an NLR > 2.49 was associated with poor postoperative prognosis (8). In vitro, neutrophils promote the invasion of HuCCT-1 (IHCC cell line) by triggering the production of hepatocyte growth factor (18). On the other 


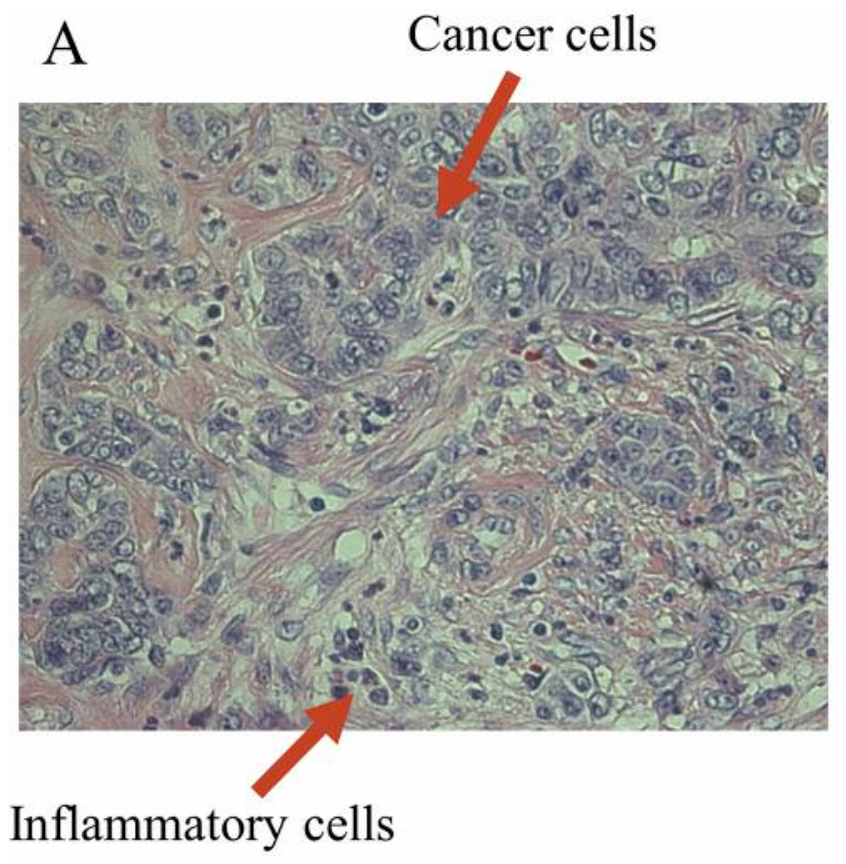

\section{$\mathrm{B}$}

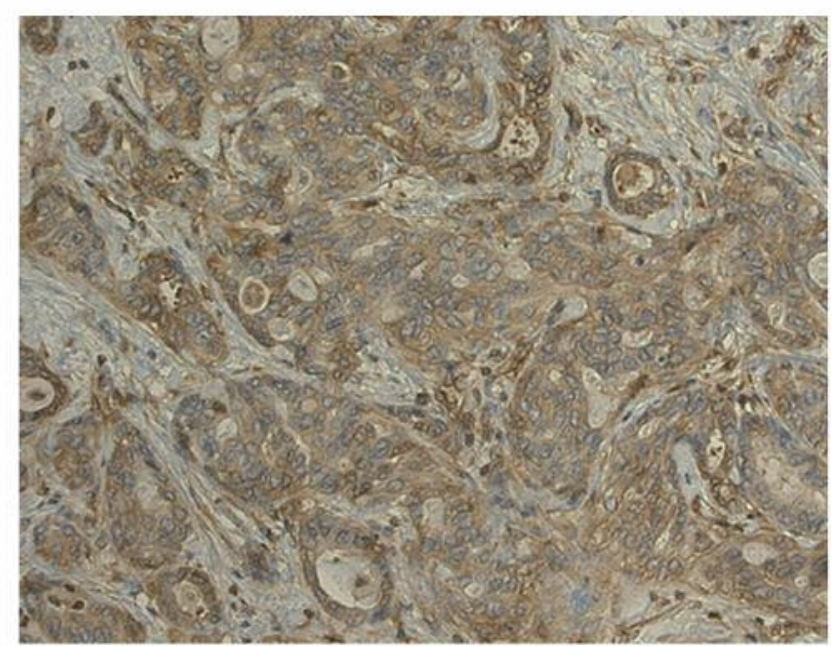

Figure 1. Histochemical analysis with hematoxylin and eosin staining of mass-forming intrahepatic cholangiocarcinoma ( $m$-IHCC) samples (A). Cancer cells and inflammatory cells are indicated with red arrows (200x). Immunohistochemical staining of NF-kb (B). All cases of m-IHCC had a high expression of $\mathrm{NF}-\mathrm{kb}(200 \times)$.

Table I. Univariate and multivariate analysis of prognostic factors for recurrence using logistic regression.

\begin{tabular}{|c|c|c|c|c|c|c|}
\hline \multirow[t]{2}{*}{ Factor } & \multicolumn{3}{|c|}{ Univariate analysis } & \multicolumn{3}{|c|}{ Multivariate analysis } \\
\hline & HR & $95 \% \mathrm{CI}$ & $p$-Value & $\mathrm{HR}$ & $95 \% \mathrm{CI}$ & $p$-Value \\
\hline Age & 0.974 & $0.90-1.05$ & 0.488 & - & - & - \\
\hline Gender (Male/Female) & 1.280 & $0.31-5.25$ & 0.729 & - & - & - \\
\hline CEA & 1.020 & $0.97-1.06$ & 0.516 & - & - & - \\
\hline CA19-9 & 1.000 & $1.00-1.00$ & 0.291 & - & - & - \\
\hline Tumor stage $(\mathrm{T} 1,2 / \mathrm{T} 3,4)$ & 3.440 & $0.92-12.8$ & 0.065 & 6.840 & $0.32-143.00$ & 0.21500 \\
\hline Lymphatic metastasis $(-/+)$ & 2.840 & $0.63-12.8$ & 0.173 & - & - & - \\
\hline Clinical stage $*(\mathrm{I}, \mathrm{II} / \mathrm{III}, \mathrm{IV})$ & 4.640 & $1.24-17.4$ & 0.023 & 1.580 & $0.08-30.90$ & 0.76400 \\
\hline SUV max & 1.070 & $0.86-1.35$ & 0.542 & - & - & - \\
\hline NLR & 1.200 & $0.84-1.72$ & 0.324 & - & - & - \\
\hline LMR & 1.020 & $0.78-1.33$ & 0.877 & - & - & - \\
\hline mGPS $(0,1,2)$ & 1.680 & $0.24-11.7$ & 0.600 & - & - & - \\
\hline Lymphocyte count & 5.090 & $1.20-21.7$ & 0.028 & 37.400 & $2.23-629.00$ & 0.01190 \\
\hline Monocyte count & 9.810 & $0.05-1800$ & 0.390 & - & - & - \\
\hline ANC & 2.650 & $1.31-5.39$ & 0.007 & 4.520 & $1.46-14.00$ & 0.00879 \\
\hline
\end{tabular}

*According to the Union for International Cancer Control (UICC) TNM staging system. Statistically significant $p$-values are shown in bold. HR, Hazard ratio; CI, confidence interval; CEA, carcinoembryonic antigen; CA19-9, cancer antigen 19-9; SUV max, maximal standardized uptake value; NLR, neutrophil-to-lymphocyte ratio; LMR, lymphocyte-to-monocyte ratio; mGPS, modified Glasgow prognostic score; ANC, absolute neutrophil count.

hand, IHCC cells were immunohistochemically shown to overexpress CXCL5, which induces neutrophil infiltration in tumor tissue (13). Recently, Zhang et al. showed that the prognostic nutritional index was inversely correlated with the systemic inflammatory response and reflected poor prognosis in patients with IHCC (19). Yoh et al. presented a new preoperative prognostic grading system for postoperative IHCC survival, which involved the NLR, c-reactive protein 

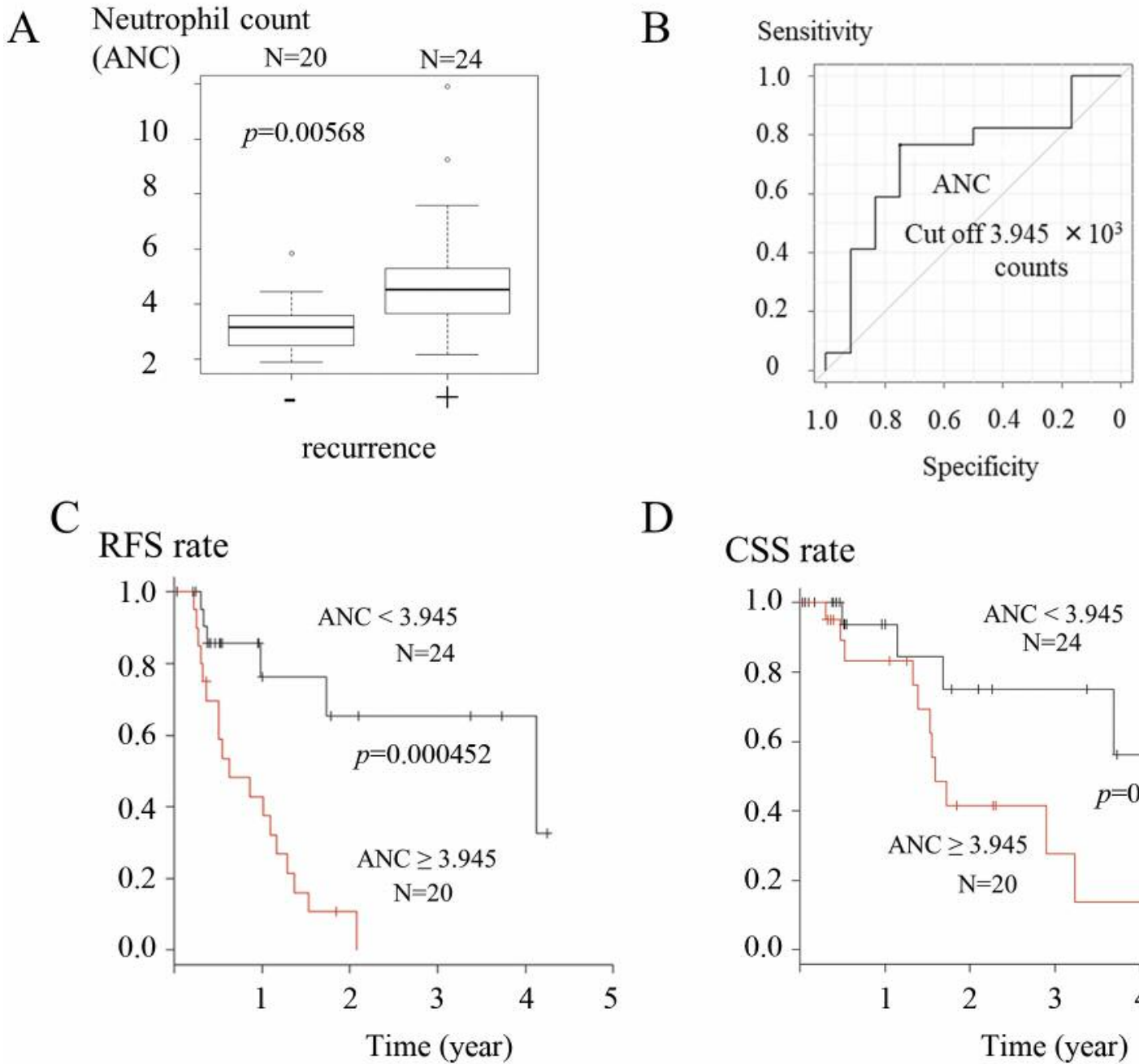

D

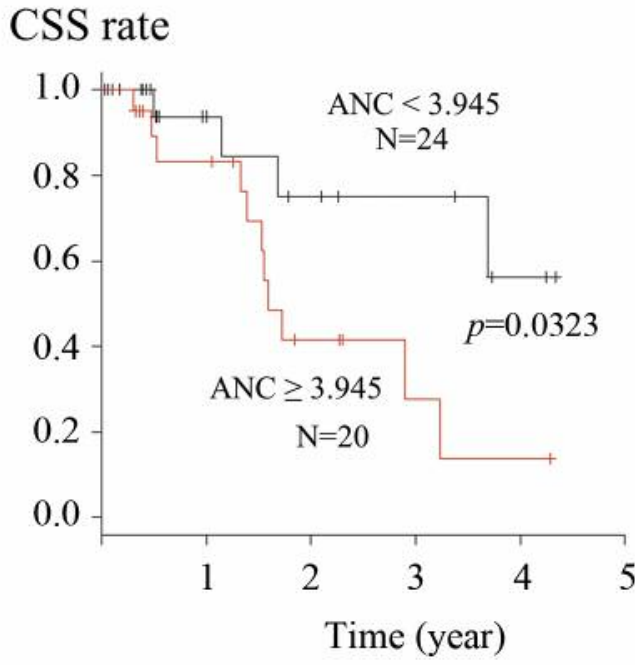

Figure 2. The absolute neutrophil count $(A N C)$ was significantly higher in the recurrence group than in the non-recurrence group ( $p=0.00568)(A)$. The ANC cut-off was determined using receiver operating characteristic curve analysis (B). The ANC cut-off value was $3.945 \times 10^{3}[$ area under the curve $(A U C)=0.721$, sensitivity=0.800, specificity=0.750]. Relationship between postoperative survival and the ANC was analyzed by KaplanMeier curve. A high ANC indicated poor prognosis for recurrence-free survival (RFS) $(p=0.00345)(C)$. A high ANC tended to indicate poor prognosis for cancer-specific survival (CSS) $(p=0.0611)(D)$.

(CRP) level, and cancer antigen 19-9 (CA19-9) level (20). These results indicate that IHCC is closely related to inflammation and suggest that IHCC cells and inflammatory cells, especially neutrophils, may mutually influence each other to promote cancer progression. The present study revealed that the preoperative ANC was significantly related to postoperative recurrence and RFS in patients with m-IHCC. In addition, local neutrophil infiltration significantly reflected the ANC. Our data support the findings of the previous reports mentioned above, suggesting that a high ANC may be a useful predictive prognostic factor in patients who undergo resection for m-IHCC.

CD66b belongs to a family of highly glycosylated CEA proteins, and has been reported as a useful marker for identifying tumor-associated neutrophils (TANs) in several cancer types $(17,21)$. In the present study, immunohistochemical analysis using the CD66b marker revealed that the ANC was correlated with neutrophil infiltration in the tumor site, and this result reflects that TANs may contribute to tumor progression of m-IHCC. We have previously reported that $\mathrm{D}$-dimer can be used as a prognostic marker in postoperative cholangiocarcinoma patients (including $\mathrm{m}$ IHCC). In the same study, D-dimer was significantly correlated with markers of systemic inflammation (22). Therefore, inflammation may have an important role in malignancy or tumor progression in patients with m-IHCC.

In literature, there is evidence supporting that FDG uptake is related to the recurrence and prognosis of resectable m- 


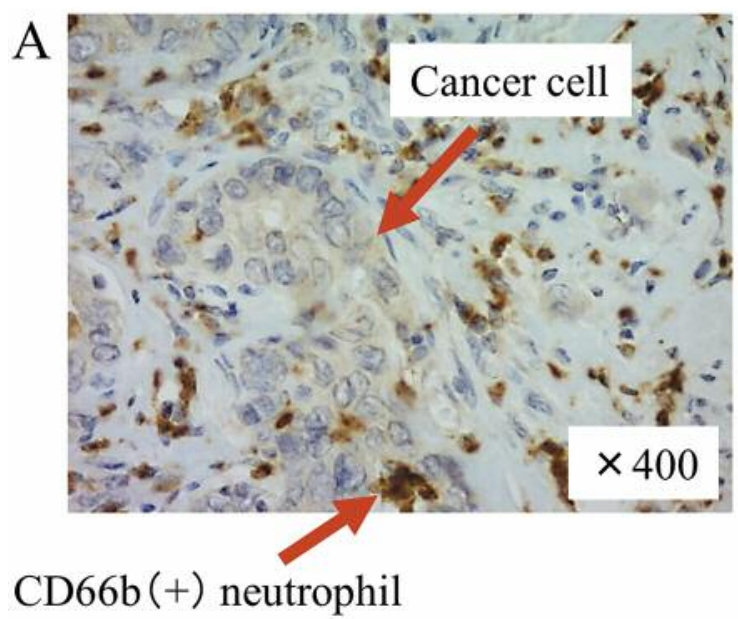

B

\begin{tabular}{|l|c|c|c|}
\hline & CD66b (-) & CD66b (+) & $p$-Value \\
\hline ANC $<3.945$ & 7 & 6 & \\
\hline ANC $\geqq 3.945$ & 1 & 10 & 0.0335 \\
\hline
\end{tabular}

Figure 3. Neutrophil infiltration in the tumor microenvironment by immunohistochemistry of CD66b in mass-forming intrahepatic cholangiocarcinoma (400x) (A). Positive correlation of the absolute neutrophil count and CD66b expression $(p=0.0355)(B)$.

IHCC. Seo et al. reported that high FDG uptake of primary lesions (SUVmax>8.5) was correlated with poor prognosis in patients with m-IHCC (23). FDG uptake in IHCC was shown to be related to glucose transporter-1 (GLUT-1) overexpression in several cancer studies $(24,25)$. Therefore, FDG uptake is considered to reflect tumor activity in patients with IHCC. Several studies have reported a correlation between FDG uptake and inflammation. Hara et al. showed that FDG uptake reflected neutrophil infiltration of a thrombus, in a murine model of deep vein thrombosis (26). Morover, El-Chemaly et al. demonstrated that fibrotic lung inflammatory cells express GLUT-1, suggesting that FDG uptake in idiopathic pulmonary fibrosis is due to the enhanced inflammatory uptake (27). With regard to cancer, Nair et al. revealed that the expression of NF-kB, a nuclear receptor that affects inflammation, was associated with FDG uptake in nonsmall cell lung cancer (28). Additionally, FDG uptake was shown to correlate with the inflammation factor hypoxiainducible factor- $1 \alpha$, in oral squamous cell carcinoma (29). In our study, the ANC was found to have a significant positive correlation with FDG uptake in patients with m-IHCC. Taken together, these findings indicate that there is a strong relationship between FDG uptake and the inflammatory status in patients with $\mathrm{m}$-IHCC. However, our study did have certain limitations, mainly due its retrospective nature and the small number of the examined cases. Therefore, further clinical studies with larger cohort sizes could fully elucidate the association of ANC with m-IHCC progression.

In conclusion, it was shown that a high ANC is significantly associated with poor prognosis in patients with m-IHCC. Therefore, the ANC may be a useful predictive biomarker for $\mathrm{m}$-IHCC recurrence and prognosis. Our findings also indicate that inflammatory factors may be promising targets for controlling cancer progression in patients with m-IHCC.
Table II. Absolute neutrophil count (ANC) and clincopathological factors.

\begin{tabular}{|c|c|c|c|}
\hline & $\begin{array}{c}\mathrm{ANC}<3.945 \\
\mathrm{n}=24\end{array}$ & $\begin{aligned} \mathrm{ANC} & \geq 3.945 \\
\mathrm{n} & =20\end{aligned}$ & $p$-Value \\
\hline \multicolumn{4}{|l|}{ Age } \\
\hline$<70$ & 8 & 4 & \multirow[t]{2}{*}{0.49800} \\
\hline$\geq 70$ & 16 & 16 & \\
\hline \multicolumn{4}{|l|}{ Gender } \\
\hline Male & 9 & 6 & \multirow[t]{2}{*}{0.75200} \\
\hline Female & 15 & 14 & \\
\hline \multicolumn{4}{|l|}{ CEA } \\
\hline$<5.0 \mathrm{ng} / \mathrm{ml}$ & 20 & 14 & \multirow[t]{2}{*}{0.47700} \\
\hline$\geq 5.0 \mathrm{ng} / \mathrm{ml}$ & 4 & 5 & \\
\hline \multicolumn{4}{|l|}{ CA19-9 } \\
\hline$<37 \mathrm{U} / \mathrm{ml}$ & 14 & 9 & \multirow[t]{2}{*}{0.54700} \\
\hline$\geq 37 \mathrm{U} / \mathrm{ml}$ & 10 & 10 & \\
\hline \multicolumn{4}{|l|}{ Tumor stage } \\
\hline $\mathrm{T} 1,2$ & 11 & 6 & \multirow[t]{2}{*}{0.34800} \\
\hline $\mathrm{T} 3,4$ & 11 & 12 & \\
\hline \multicolumn{4}{|c|}{ Lymphatic metastasis } \\
\hline- & 18 & 13 & \multirow[t]{2}{*}{0.50400} \\
\hline+ & 5 & 6 & \\
\hline \multicolumn{4}{|l|}{ Clinical stage* } \\
\hline I, II & 11 & 5 & \multirow[t]{2}{*}{0.21300} \\
\hline III, IV & 13 & 15 & \\
\hline \multicolumn{3}{|l|}{ mGPS } & \multirow[t]{4}{*}{0.11000} \\
\hline 0 & 23 & 15 & \\
\hline 1 & 1 & 2 & \\
\hline 2 & 0 & 3 & \\
\hline \multicolumn{3}{|l|}{ NLR } & \multirow[t]{3}{*}{0.01120} \\
\hline$<3.0$ & 20 & 9 & \\
\hline$\geq 3.0$ & 4 & 11 & \\
\hline \multicolumn{4}{|l|}{$\mathrm{SUV}_{\max }$} \\
\hline$<10.0$ & 21 & 9 & \multirow[t]{2}{*}{0.00089} \\
\hline$\geq 10.0$ & 1 & 10 & \\
\hline
\end{tabular}

*According to the Union for International Cancer Control (UICC) TNM staging system. Statistically significant $p$-values are shown in bold. CEA, Carcinoembryonic antigen; CA19-9, cancer antigen 19-9; mGPS, modified Glasgow prognostic score; NLR, neutrophil-to-lymphocyte ratio; $\mathrm{SUV}_{\max }$, maximal standardized uptake value. 
Table III. Univariate and multivariate analysis of prognostic factors for recurrence-free survival.

\begin{tabular}{|c|c|c|c|c|c|c|}
\hline \multirow[t]{2}{*}{ Factor } & \multicolumn{3}{|c|}{ Univariate analysis } & \multicolumn{3}{|c|}{ Multivariate analysis } \\
\hline & HR & $95 \% \mathrm{CI}$ & $p$-Value & HR & $95 \% \mathrm{CI}$ & $p$-Value \\
\hline Age $(\leq 65 />65)$ & 1.385 & $0.54-3.54$ & 0.4961 & - & - & - \\
\hline Gender $(\mathrm{M} / \mathrm{F})$ & 1.038 & $0.44-2.46$ & 0.9318 & - & - & - \\
\hline $\mathrm{CEA}(<5.0 / \geq 5.0)$ & 2.566 & $0.88-7.45$ & 0.08391 & 2.035 & $0.40-10.3$ & 0.39100 \\
\hline CA19-9 $(<37 / \geq 37)$ & 1.242 & $0.55-2.83$ & 0.605 & - & - & - \\
\hline Tumor stage $(\mathrm{T} 1,2 / \mathrm{T} 3,4)$ & 1.352 & $0.51-3.57$ & 0.5437 & - & - & - \\
\hline Lymphatic metastasis $(-/+)$ & 5.755 & $1.91-17.3$ & 0.002 & 6.410 & $1.51-27.2$ & 0.01180 \\
\hline Clinical stage* (I, II/III, IV) & 1.928 & $0.71-5.22$ & 0.197 & - & - & - \\
\hline NL ratio $(<3.0 / \geq 3.0)$ & 1.579 & $0.70-3.57$ & 0.2724 & - & - & - \\
\hline mGPS $(0 / 1,2)$ & 2.545 & $0.93-6.93$ & 0.06762 & 0.883 & $0.16-4.93$ & 0.88700 \\
\hline $\operatorname{SUV}_{\max }($ cut off 10.0$)$ & 3.019 & $1.27-7.19$ & 0.01259 & 1.609 & $0.41-6.28$ & 0.49400 \\
\hline ANC $(<3.945 / \geq 3.945)$ & 5.144 & $1.87-14.1$ & 0.001478 & 5.105 & $1.57-16.6$ & 0.00678 \\
\hline
\end{tabular}

*According to the Union for International Cancer Control (UICC) TNM staging system. Statistically significant $p$-values are shown in bold. HR, Hazard ratio; CI, confidence interval; CEA, carcinoembryonic antigen; CA19-9, cancer antigen 19-9; NLR, neutrophil-to-lymphocyte ratio; mGPS, modified Glasgow prognostic score; $\mathrm{SUV}_{\max }$, maximal standardized uptake value.

Table IV. Univariate and multivariate analysis of prognostic factors for cancer-specific survival.

\begin{tabular}{|c|c|c|c|c|c|c|}
\hline \multirow[t]{2}{*}{ Factor } & \multicolumn{3}{|c|}{ Univariate analysis } & \multicolumn{3}{|c|}{ Multivariate analysis } \\
\hline & HR & $95 \% \mathrm{CI}$ & $p$-Value & HR & $95 \% \mathrm{CI}$ & $p$-Value \\
\hline Age $(\leq 65 />65)$ & 1.014 & $0.34-2.99$ & 0.9801 & - & - & - \\
\hline Gender $(\mathrm{M} / \mathrm{F})$ & 1.349 & $0.46-3.99$ & 0.5876 & - & - & - \\
\hline CEA $(<5.0 / \geq 5.0)$ & 3.988 & $1.16-13.7$ & 0.02833 & 1.908 & $0.49-7.32$ & 0.3470 \\
\hline CA19-9 $(<37 / \geq 37)$ & 0.9992 & $0.35-2.89$ & 0.9988 & - & - & - \\
\hline Tumor stage $(\mathrm{T} 1,2 / \mathrm{T} 3,4)$ & 1.814 & $0.50-6.61$ & 0.3665 & - & - & - \\
\hline Lympatic metastasis $(-/+)$ & 3.692 & $1.27-10.7$ & 0.017 & 3.950 & $1.17-13.2$ & 0.0262 \\
\hline Clinical stage* (I, II/III, IV) & 1.794 & $0.51-6.38$ & 0.3661 & - & - & - \\
\hline NL ratio $(<3.0 / \geq 3.0)$ & 1.691 & $0.61-4.68$ & 0.3119 & - & - & - \\
\hline mGPS $(0 / 1,2)$ & 2.244 & $0.71-7.08$ & 0.1677 & - & - & - \\
\hline $\mathrm{SUV}_{\max }($ cut off 10.0$)$ & 1.992 & $0.65-6.12$ & 0.2283 & - & - & - \\
\hline $\operatorname{ANC}(<3.945 / \geq 3.945)$ & 3.366 & $1.04-10.8$ & 0.04233 & 3.210 & $0.88-11.7$ & 0.0770 \\
\hline
\end{tabular}

*According to the Union for International Cancer Control (UICC) TNM staging system. Statistically significant $p$-values are shown in bold. HR, Hazard ratio; CI, confidence interval; CEA, carcinoembryonic antigen; CA19-9, cancer antigen 19-9; NLR, neutrophil-to-lymphocyte ratio; mGPS, modified Glasgow prognostic score; $\mathrm{SUV}_{\max }$, maximal standardized uptake value; ANC, absolute neutrophil count.

\section{Acknowledgements}

Authors would like to thank Misato Negishi, and Ms. Tomoko Ubukata for their excellent assistance.

\section{References}

1 Simo KA, Halpin LE, McBrier NM, Hessey JA, Baker E, Ross S, Swan RZ, Iannitti DA and Martinie JB: Multimodality treatment of intrahepatic cholangiocarcinoma: A review. J Surg Oncol 113(1): 62-83, 2016.

2 Yamamoto $\mathrm{M}$ and Ariizumi S: Surgical outcomes of intrahepatic cholangiocarcinoma. Surg Today 41(7): 896-902, 2011.
3 Minami S, Ihara S, Kim SH, Yamamoto S and Komuta K: Lymphocyte to monocyte ratio and modified glasgow prognostic score predict prognosis of lung adenocarcinoma without driver mutation. World J Oncol 9(1): 13-20, 2018.

4 Okano N, Kasuga A, Kawai K, Yamauchi Y, Kobayashi T, Naruge D, Nagashima F and Furuse J: The modified glasgow prognostic score in patients with gemcitabine-refractory biliary tract cancer. Anticancer Res 38(3): 1755-1761, 2018.

5 Ying HQ, Deng QW, He BS, Pan YQ, Wang F, Sun HL, Chen J, Liu $X$ and Wang SK: The prognostic value of preoperative nlr, d-nlr, plr and lmr for predicting clinical outcome in surgical colorectal cancer patients. Med Oncol 31(12): 305, 2014.

6 Yamanaka T, Matsumoto S, Teramukai S, Ishiwata R, Nagai Y and Fukushima M: The baseline ratio of neutrophils to 
lymphocytes is associated with patient prognosis in advanced gastric cancer. Oncology 73(3-4): 215-220, 2007.

7 Lin G, Liu Y, Li S, Mao Y, Wang J, Shuang Z, Chen J and Li S: Elevated neutrophil-to-lymphocyte ratio is an independent poor prognostic factor in patients with intrahepatic cholangiocarcinoma. Oncotarget 7(32): 50963-50971, 2016.

8 Chen Q, Yang LX, Li XD, Yin D, Shi SM, Chen EB, Yu L, Zhou ZJ, Zhou SL, Shi YH, Fan J, Zhou J and Dai Z: The elevated preoperative neutrophil-to-lymphocyte ratio predicts poor prognosis in intrahepatic cholangiocarcinoma patients undergoing hepatectomy. Tumour Biol 36(7): 5283-5289, 2015

9 Wang Z, Sheng YY, Dong QZ and Qin LX: Hepatitis b virus and hepatitis c virus play different prognostic roles in intrahepatic cholangiocarcinoma: A meta-analysis. World J Gastroenterol 22(10): 3038-3051, 2016.

10 Jeong S, Tong Y, Sha M, Gu J and Xia Q: Hepatitis b virusassociated intrahepatic cholangiocarcinoma: A malignancy of distinctive characteristics between hepatocellular carcinoma and intrahepatic cholangiocarcinoma. Oncotarget 8(10): 17292-17300, 2017.

11 Kim HJ, Kim JS, Joo MK, Lee BJ, Kim JH, Yeon JE, Park JJ, Byun KS and Bak YT: Hepatolithiasis and intrahepatic cholangiocarcinoma: A review. World J Gastroenterol 21(48): 13418-13431, 2015.

12 Jaiswal M, LaRusso NF, Burgart LJ and Gores GJ: Inflammatory cytokines induce DNA damage and inhibit DNA repair in cholangiocarcinoma cells by a nitric oxide-dependent mechanism. Cancer Res 60(1): 184-190, 2000.

13 Zhou SL, Dai Z, Zhou ZJ, Chen Q, Wang Z, Xiao YS, Hu ZQ, Huang XY, Yang GH, Shi YH, Qiu SJ, Fan J and Zhou J: Cxcl5 contributes to tumor metastasis and recurrence of intrahepatic cholangiocarcinoma by recruiting infiltrative intratumoral neutrophils. Carcinogenesis 35(3): 597-605, 2014.

14 Gu FM, Gao Q, Shi GM, Zhang X, Wang J, Jiang JH, Wang XY, Shi YH, Ding ZB, Fan J and Zhou J: Intratumoral il-17(+) cells and neutrophils show strong prognostic significance in intrahepatic cholangiocarcinoma. Ann Surg Oncol 19(8): 25062514, 2012.

15 Kanda Y: Investigation of the freely available easy-to-use software 'ezr' for medical statistics. Bone Marrow Transplant 48(3): 452-458, 2013.

16 Cardinale V, Bragazzi MC, Carpino G, Torrice A, Fraveto A, Gentile R, Pasqualino V, Melandro F, Aliberti C, Bastianelli C, Brunelli R, Berloco PB, Gaudio E and Alvaro D: Cholangiocarcinoma: Increasing burden of classifications. Hepatobiliary Surg Nutr 2(5): 272-280, 2013.

17 Zhou SL, Zhou ZJ, Hu ZQ, Huang XW, Wang Z, Chen EB, Fan J, Cao Y, Dai Z and Zhou J: Tumor-associated neutrophils recruit macrophages and t-regulatory cells to promote progression of hepatocellular carcinoma and resistance to sorafenib. Gastroenterology 150(7): 1646-1658e1617, 2016.

18 Imai Y, Kubota Y, Yamamoto S, Tsuji K, Shimatani M, Shibatani N, Takamido S, Matsushita M and Okazaki K: Neutrophils enhance invasion activity of human cholangiocellular carcinoma and hepatocellular carcinoma cells: An in vitro study. J Gastroenterol Hepatol 20(2): 287-293, 2005.

19 Zhang C, Wang H, Ning Z, Xu L, Zhuang L, Wang P and Meng $\mathrm{Z}$ : Prognostic nutritional index serves as a predictive marker of survival and associates with systemic inflammatory response in metastatic intrahepatic cholangiocarcinoma. Onco Targets Ther 9: 6417-6423, 2016.

20 Yoh T, Seo S, Hatano E, Taura K, Fuji H, Ikeno Y, Okuda Y, Yasuchika K, Kaido T, Okajima $\mathrm{H}$ and Uemoto S: A novel biomarker-based preoperative prognostic grading system for predicting survival after surgery for intrahepatic cholangiocarcinoma. Ann Surg Oncol 24(5): 1351-1357, 2017.

21 Jensen HK, Donskov F, Marcussen N, Nordsmark M, Lundbeck $\mathrm{F}$ and von der Maase $\mathrm{H}$ : Presence of intratumoral neutrophils is an independent prognostic factor in localized renal cell carcinoma. J Clin Oncol 27(28): 4709-4717, 2009.

22 Watanabe A, Araki K, Hirai K, Kubo N, Igarashi T, Tsukagoshi M, Ishii N, Hoshino K, Kuwano H and Shirabe K: A novel clinical factor, d-dimer platelet multiplication, may predict postoperative recurrence and prognosis for patients with cholangiocarcinoma. Ann Surg Oncol 23(Suppl 5): 886-891, 2016.

23 Seo S, Hatano E, Higashi T, Nakajima A, Nakamoto Y, Tada M, Tamaki N, Iwaisako K, Mori A, Doi R, Ikai I and Uemoto S: Fluorine-18 fluorodeoxyglucose positron emission tomography predicts lymph node metastasis, p-glycoprotein expression, and recurrence after resection in mass-forming intrahepatic cholangiocarcinoma. Surgery 143(6): 769-777, 2008.

24 Paudyal B, Oriuchi N, Paudyal P, Tsushima Y, Higuchi T, Miyakubo M, Ishikita $\mathrm{T}$, Nakajima $\mathrm{T}$ and Endo $\mathrm{K}$ : Clinicopathological presentation of varying $18 \mathrm{f}$-fdg uptake and expression of glucose transporter 1 and hexokinase ii in cases of hepatocellular carcinoma and cholangiocellular carcinoma. Ann Nucl Med 22(1): 83-86, 2008.

25 Suzuki H, Komuta M, Bolog A, Yokobori T, Wada S, Araki K, Kubo N, Watanabe A, Tsukagoshi M and Kuwano H: Relationship between 18-f-fluoro-deoxy-d-glucose uptake and expression of glucose transporter 1 and pyruvate kinase $\mathrm{m} 2$ in intrahepatic cholangiocarcinoma. Dig Liver Dis 47(7): 590-596, 2015.

26 Hara T, Truelove J, Tawakol A, Wojtkiewicz GR, Hucker WJ, MacNabb MH, Brownell AL, Jokivarsi K, Kessinger CW, Jaff MR, Henke PK, Weissleder $R$ and Jaffer FA: 18ffluorodeoxyglucose positron emission tomography/computed tomography enables the detection of recurrent same-site deep vein thrombosis by illuminating recently formed, neutrophil-rich thrombus. Circulation 130(13): 1044-1052, 2014.

27 El-Chemaly S, Malide D, Yao J, Nathan SD, Rosas IO, Gahl WA, Moss $\mathrm{J}$ and Gochuico BR: Glucose transporter-1 distribution in fibrotic lung disease: Association with [(1)(8)f]2-fluoro-2-deoxyglucose-pet scan uptake, inflammation, and neovascularization. Chest 143(6): 1685-1691, 2013.

28 Nair VS, Gevaert O, Davidzon G, Plevritis SK and West R: Nfkappab protein expression associates with (18)f-fdg pet tumor uptake in non-small cell lung cancer: A radiogenomics validation study to understand tumor metabolism. Lung Cancer 83(2): 189196, 2014.

29 Sato J, Kitagawa Y, Yamazaki Y, Hata H, Okamoto S, Shiga T, Shindoh M, Kuge Y and Tamaki N: ${ }^{18}$ f-fluoromisonidazole pet uptake is correlated with hypoxia-inducible factor-1alpha expression in oral squamous cell carcinoma. J Nucl Med 54(7): 1060-1065, 2013. 